\title{
IMPLICACIONES ÉTICAS Y SOCIALES DE LA INTRODUCCIÓN DE LA VACUNA CONTRA EL VIRUS DEL PAPILOMA HUMANO EN MÉXICO: REFLEXIONES SOBRE UNA PROPUESTA DE INTERVENCIÓN
}

\author{
Alejandra Prieto de la Rosa*, Cristina Gutiérrez Delgado**, Dafna Feinholz Klip***, \\ Gisela Morales González*** y Renee Witlen****
}

\begin{abstract}
Resumen: El objetivo de este trabajo es identificar aspectos éticos y sociales relacionados con la introducción de la vacuna contra el virus del papiloma humano (VPH) en México para una decisión informada y justa que considere no sólo los aspectos financieros.

De acuerdo con la información sobre eficiencia y costo de la vacuna, su introducción al sistema público mexicano puede acarrear consecuencias no previstas para las participantes y para toda la sociedad, por lo que debe examinarse en el contexto mexicano, reconociendo sus riesgos sin sobrestimar resultados comprobados o potenciales derivados de diversas políticas de vacunación. Exponer la evidencia científica y los motivos para la toma de decisiones es esencial. También, dirigirse de manera responsable hacia una decisión política sobre la implementación de programas de vacunación, tamizaje y tratamiento de cáncer cervical con base en el potencial para salvar vidas que tienen las nuevas tecnologías, siempre que se respeten los derechos, opiniones y preocupaciones de las mujeres a las que va dirigida la vacuna contra el VPH y que representen un valor agregado para la sociedad mexicana en su conjunto.
\end{abstract}

Palabras clave: cáncer cervical, vacuna VPH, ética, toma de decisiones

\section{ETHICAL AND SOCIAL IMPLICATIONS OF THE INTRODUCTION OF THE VACCINE AGAINST THE HUMAN PAPILLOMA VIRUS IN MEXICO: REFLECTIONS ON A PROPOSED INTERVENTION}

\begin{abstract}
The objective of this work is to identify ethical and social aspects related to the introduction of the vaccine against the human papillomavirus (HPV) in Mexico for an informed and just decision that considers not only financial aspects. According to information about the efficiency and cost of the vaccine, its introduction to the public health system of Mexico can lead to unforeseen consequences for the participants and for society as a whole. The introduction of the vaccine should examine the Mexican context, recognizing its risks without overestimating potential or proven results derived from diverse vaccine policies. Exposing scientific evidence and the motives for decision making is essential. Also, it is essential to take a responsible stand toward political decisions on the implementation of vaccine programs, screening and treatment for cervical cancer based on the potential for new technologies to save lives, always with respect for the rights, opinions and preoccupations of women, the recipients of the HPV vaccine, and that represent an added value for Mexican society as a whole.
\end{abstract}

Key words: cervical cancer, HPV vaccine, ethics, decision making

\section{IMPLICAÇÓES ÉTICAS E SOCIAIS DA INTRODUÇÃO DA VACINA CONTRA O VIRUS DO PAPILOMA HUMANO NO MÉXICO: REFLEXÓES SOBRE UMA PROPOSTA DE INTERVENÇÁO}

Resumo: O objetivo deste trabalho é identificar aspectos éticos e sociais relacionados com a introdução da vacina contra o vírus do papiloma humano (HPV) no México para uma decisão informada e justa que considere não somente os aspectos financeiros.

De acordo com a informação sobre eficiência e custo da vacina, sua introdução no sistema público mexicano pode carrear conseqüências não previstas para as participantes e para toda a sociedade, pelo que deve ser examinada no contexto mexicano, reconhecendo seus riscos sem superestimar resultados comprovados ou potenciais derivados de diversas políticas de vacinação. Expor a evidência científica e os motivos para a tomada de decisões é essencial. Também, dirigir-se de maneira responsável para uma decisão política sobre a implementação de programas de vacinação, tamisaçáo e tratamento de câncer cervical com base no potencial para salvar vidas que têm as novas tecnologias, sempre que se respeitam direitos, opinióes e preocupaçóes das mulheres às quais é destinada a vacina contra o HPV e que representam um valor agregado para a sociedade mexicana em seu conjunto.

Palavras-chave: câncer cervical, vacina HPV, ética, tomada de decisóes

\footnotetext{
* Centro Nacional de Excelencia Tecnológica en Salud, Secretaría de Salud, México

Correspondencia: alejandraprieto@salud.gob.mx

** Unidad de Análisis Económico, Secretaría de Salud, México

*** Comisión Nacional de Bioética, Secretaría de Salud, México

**** Escuela de Medicina de Harvard, Universidad de Harvard, EEUU
} 


\section{Introducción}

La idea de incorporar la vacuna contra el virus del papiloma humano (VPH) en México surge de las recomendaciones sobre su uso en servicios públicos de la Organización Mundial de la Salud y de distintos países desarrollados (tabla 1). Dichos países cuentan con exitosos programas nacionales de tamizaje para cáncer cervical $(\mathrm{CaCU})$, por lo que su preocupación actual es determinar el impacto marginal que la introducción de la vacuna tendrá en los casos potencialmente evitados tanto en morbilidad como en mortalidad.

En 2007 el Centro Nacional de Equidad de Género y Salud Reproductiva de la Secretaría de Salud presentó una propuesta ${ }^{1}$ para incorporar nuevas tecnologías de prevención, tamizaje y control del $\mathrm{CaCU}$ en la que se incluyó la vacuna contra $\mathrm{VPH}^{2}$.

En septiembre del mismo ańo, en el Senado de la República, se presentó un punto de acuerdo(1) de asignación de recursos etiquetados a un programa de vacunación contra el CaCU, y el Presupuesto de Egresos de la Federación 2008(2) ya incluye una partida destinada -entre otros rubros- al programa piloto de la vacuna contra VPH. Se realizó un análisis de costo-efectividad generalizado para determinar la combinación más costo-efectiva de tres intervenciones preventivas contra el $\mathrm{CaCU}$ para el sector salud en México: tamiz por Papanicolaou, tamiz por captura de híbridos y vacuna contra VPH(3). Dicho análisis respondió parcialmente a dos preguntas básicas para una política pública: ante un presupuesto limitado, ¿̇cuál es la combinación de intervenciones que maximiza los resultados en salud?; ¿cuál sería el impacto en el gasto de la introducción de nuevas tecnologías?

Los escenarios planteados para la introducción de la vacuna contra VPH fueron: a) plan piloto de vacunación al 10\% de las jóvenes de 12 años; b) vacunación universal -con cobertura del $80 \%$ - a mujeres jóvenes de 12 años; c) plan piloto con tamizaje, y d) vacunación universal con tamizaje. Cada escenario, con sus respectivos resultados, tiene implicaciones éticas, sociales y culturales importantes.

Los aspectos fundamentales para el presente análisis -y que forman el común denominador de los escenarios descritos- son: efectividad probada de la vacuna; población objetivo, grupo de edad, criterios de financiamiento, estrategia de implementación y consideraciones generales sobre la vacuna.

En un proceso justo de toma de decisiones(4) se requiere que antes de cualquier cambio en el ámbito público se realice un análisis detallado de aspectos financieros y no financieros de la política que se desea implementar, hasta obtener un consenso para determinar la conveniencia y las repercusiones operativas del posible cambio en la organización de los recursos.

En este caso se deben considerar las repercusiones éticas y sociales de las intervenciones analizadas como un componente clave en un proceso de toma de decisión más transparente, incluyente y consensuado. Debe ser una política pública basada en las condiciones de salud, económicas y sociales de la población en México. Además, presentar argumentos aceptables para todos los actores involucrados y accesibles para el público en general(4).

1 Centro Nacional de Equidad de Género y Salud Reproductiva (marzo 2007). Propuesta de mejora del programa de prevención y control del $\mathrm{CaCU}$. Secretaría de Salud (documento no publicado).

2 La propuesta se presentó antes de la publicación de la más reciente actualización a la norma oficial mexicana "Sobre el control y tratamiento del cáncer cérvico-uterino" (NOM-014-SSA2-1994). Dicha actualización, trabajada desde 2004, incluye la vacuna como parte de las acciones que pueden desarrollarse en el Programa Nacional de Detección Oportuna de $\mathrm{CaCu}$. 
Tabla 1. Recomendaciones internacionales del uso de la vacuna VPH en servicios públicos de salud

\begin{tabular}{|c|c|c|c|c|c|}
\hline País u Organización ${ }^{3}$ & $\begin{array}{c}\text { Edad recomendada } \\
\text { para aplicar la } \\
\text { vacuna }\end{array}$ & $\begin{array}{c}\text { Extensión de la } \\
\text { aplicación }\end{array}$ & Tamiz recomendado & \begin{tabular}{|c|} 
Fecha \\
publicación de la \\
recomendación
\end{tabular} & Otras observaciones \\
\hline $\begin{array}{l}\text { Organización Mundial } \\
\text { de la Salud (OMS) } \\
\text { (1) }\end{array}$ & $\begin{array}{l}\text { Grupo prioritario: } \\
\text { niñas de } 9 \text { a } 13 \\
\text { ańos }\end{array}$ & $\begin{array}{l}\text { Grupo de } \\
\text { "repesca": 14- } \\
26 \text { años }\end{array}$ & $\begin{array}{l}\text { Ninguno en } \\
\text { particular, depende } \\
\text { de recursos en cada } \\
\text { país }\end{array}$ & 2006 & $\begin{array}{l}\text { Para introducción al esquema } \\
\text { de vacunación considerar } \\
\text { costo, beneficios indirectos y } \\
\text { sensibilización enfocados al grupo } \\
\text { prioritario }\end{array}$ \\
\hline $\begin{array}{l}\text { Espańa Consenso de } \\
\text { Sociedades Científicas } \\
\text { (2) }\end{array}$ & $\begin{array}{l}\text { Niñas de } 9 \text { a } 13 \\
\text { años }\end{array}$ & 14 a 26 ańos & $\begin{array}{l}\text { Captura híbrida } \\
\text { para edades mayores } \\
\text { a } 35\end{array}$ & Enero 31, 2007 & $\begin{array}{l}\text { Sugiere adecuar tiempos del } \\
\text { programa de tamizaje }\end{array}$ \\
\hline $\begin{array}{l}\text { Estados Unidos } \\
\text { Advisory Committee on } \\
\text { Immunization Practices } \\
\text { (3) }\end{array}$ & $\begin{array}{l}\text { Niñas de } 11 \text { a } 12 \\
\text { años }\end{array}$ & 13 a 26 ańos & $\begin{array}{l}\text { Papanicolao y } \\
\text { prueba ADN para } \\
\text { edades mayores a } 30\end{array}$ & Marzo 23, 2007 & $\begin{array}{l}\text { Recomienda realizar } \\
\text { actualizaciones de evaluación } \\
\text { económica de la vacuna y su } \\
\text { relación con el tamizaje }\end{array}$ \\
\hline $\begin{array}{l}\text { Canadá } \\
\text { Health Canada National } \\
\text { Advisory Committee on } \\
\text { Immunization } \\
(4)\end{array}$ & $\begin{array}{l}\text { Niñas de } 9 \text { a } 13 \\
\text { años }\end{array}$ & $\begin{array}{l}\text { No recomienda } \\
\text { ninguna } \\
\text { extensión }\end{array}$ & $\begin{array}{l}\text { Papanicolao y } \\
\text { prueba ADN para } \\
\text { edades mayores a } 30\end{array}$ & Febrero 2007 & \\
\hline $\begin{array}{l}\text { Australia } \\
(5)\end{array}$ & $\begin{array}{l}\text { Mujeres en el } \\
\text { primer ańo de } \\
\text { bachillerato y } \\
\text { hombres de 9 a } 15 \\
\text { años }\end{array}$ & $\begin{array}{l}\text { Cobertura } \\
\text { en el periodo } \\
2006-2010 \text { de } \\
\text { mujeres de } 9 \text { a } \\
26 \text { ańos }\end{array}$ & Papanicolao & Noviembre 2006 & $\begin{array}{l}\text { Se están realizando estudios } \\
\text { de costo-efectividad. Se aceptó } \\
\text { financiar la vacuna tras una } \\
\text { negociación con el productor para } \\
\text { reducir el precio. }\end{array}$ \\
\hline $\begin{array}{l}\text { Reino Unido } \\
\text { (6) }\end{array}$ & Sin recomendación & $\begin{array}{l}\text { Sin } \\
\text { recomendación }\end{array}$ & $\begin{array}{l}\text { Papanicolao y } \\
\text { prueba ADN para } \\
\text { edades mayores a } 30\end{array}$ & $\begin{array}{l}\text { Mayo 22, } 2006 \\
\text { y septiembre, } 22 \\
2006\end{array}$ & $\begin{array}{l}\text { Evaluación económica en proceso } \\
\text { por Imperial Collegue }\end{array}$ \\
\hline $\begin{array}{l}\text { Dinamarca } \\
(7)\end{array}$ & $\begin{array}{l}12 \text { años, en } \\
\text { discusión si se } \\
\text { aplica a ambos } \\
\text { sexos }\end{array}$ & $\begin{array}{l}\text { Sin } \\
\text { recomendación }\end{array}$ & Papanicolao & Mayo 2007 & $\begin{array}{l}\text { Los datos provienen de una } \\
\text { evaluación tecnológica. No existe } \\
\text { aún recomendación oficial del } \\
\text { Danish National Board of Health }\end{array}$ \\
\hline $\begin{array}{l}\text { México } \\
\text { CNEGYSR } \\
\text { (8) }\end{array}$ & $\begin{array}{l}\text { Niñas de } 12 \text { años } \\
\text { (propuesta de } \\
\text { prueba piloto a } \\
100,000 \text { niñas) }\end{array}$ & $\begin{array}{l}\text { Sin } \\
\text { recomendación }\end{array}$ & $\begin{array}{l}\text { Papanicolao según } \\
\text { norma (22) y CH2 }\end{array}$ & Marzo 20, 2007 & $\begin{array}{l}\text { Solicitó evaluación económica de } \\
\text { la vacuna y tamiz por } \mathrm{CH} 2 \text { a la } \\
\text { Unidad de Análisis Económico de } \\
\text { la Secretaría de Salud }\end{array}$ \\
\hline
\end{tabular}

3 Fuentes:

(1) Organización Mundial de la Salud. Preparación de la introducción de las vacunas contra el virus del papiloma humano, orientaciones normativas y programáticas para los países. OMS: 2006.

(2) Sociedades Científicas de Espańa. Vacunas profilácticas frente al VPH. Documento de consenso. 2007

(3) American Cancer Society. American Cancer Society Guideline for Human Papillomavirus (HPV) vaccine use to prevent cervical cancer and its precursors: 2007. Disponible en http://caonline.amcancersoc.org/egi/content/full/57/1/7

(4) Canadian Medical Association. Debate begins over public funding for HPV vaccine. Canadian Medical Association Journal March, $27,2007$. Disponible en http://www.cmaj.ca/cgi/content/full/176/7/913

(5) Department of Health and Ageing. Australian Government funding of Gardasil. Australian Government; November $28,2006$.

(6) Join Committee on Vaccination and Immunisation. Modelling the cost-effectiveness of HPV vaccine JCVI/HPV(06)3. United Kingdom: Department of Health. Disponible en www.advisorybodis.doh.gov.uk/jcvi

(7) National Board of Health, Danish Centre for Health Technology Assessment. Reduction in the risk of cervical cancer by vaccination against human papillomavirus (HPV). Health technology assessment 2007; 9(1).

(8) Centro Nacional de Equidad de Género y Salud Reproductiva. Propuesta de mejora del programa de prevención y control del CaCu. México: Secretaría de Salud; marzo 2007 (mimeo). 


\section{Evidencia sobre efectividad}

Los resultados sobre efectividad clínica disponibles deben ser analizados tomando en consideración la calidad y cantidad de evidencia científica utilizada. Si bien la vacuna contra VPH potencialmente inmunizaría a las mujeres ante los tipos 16 y 18 que se vinculan con $70 \%$ de los casos del CaCU, existe al menos 30\% de casos no cubiertos por la vacuna que requerirán ser detectados, idealmente en forma temprana, mediante tamizaje.

Una revisión de literatura -incluyendo la presentada por los fabricantes de la vacuna- revela un importante número de preguntas sin responder. La información sobre los efectos y la protección que proporciona la vacuna contra VPH es aún incierta. A la fecha sólo se encuentran disponibles algunos estudios aleatorios controlados que proveen la evidencia de calidad existente $(5,6,7)$. La relativamente larga fase de inducción de 20 a 25 ańos entre la exposición al agente productor (VPH) y el desarrollo del CaCU es un reto que los investigadores enfrentan para producir evidencia en corto tiempo. En los estudios se usan resultados intermedios clave -lesiones cervicales de alto grado asociadas a los tipos 16 y 18 de VPH- con el fin de que los datos puedan presentarse de manera oportuna(6). Sin embargo, se requiere evidencia de estudios de largo plazo que correlacionen el uso de la vacuna y sus efectos en la prevención del $\mathrm{CaCU}$ como resultado final.

Hasta ahora la vacuna ha sido probada en estudios con un máximo de cinco ańos de seguimiento; por ello, es necesario monitorear su efectividad a largo plazo mediante un registro de datos clínicos y administrativos para determinar si se requieren dosis de refuerzo y los efectos adversos que podrían surgir al aplicarse a grandes grupos de población $(8,9)$.

Una revisión sistemática(10) encuentra que la vacuna es altamente eficaz en el corto plazo -sobre todo si los resultados clínicos se analizan en conjunto-, aunque señala debilidades metodológicas en los reportes de los estudios revisados, lo que -combinado con los escasos datos disponibles- sigue dejando lagunas en la información. Esta situación no es inusual durante el desarrollo de nuevos productos farmacéuticos; sin embargo, previene y alerta contra la formulación de juicios demasiado optimistas sobre los beneficios y la minimización de los riesgos potenciales de la vacuna.

Lo anterior es relevante para establecer una política pública como la que ahora se enfrenta, en la cual la tecnología es elaborada por sólo dos compañías activamente involucradas en la generación de información crítica sobre la efectividad y el costo-efectividad de su producto $(11)^{4}$.

¿Por qué invertir recursos públicos para financiar una intervención que aún plantea dudas sobre su efectividad a largo plazo, cuando los programas internacionales exitosos para reducir la incidencia de $\mathrm{CaCU}$ se han basado en estrategias mejoradas de tamizaje, seguimiento y tratamiento?

Algunos autores sugieren que -en países en vías de desarrollo- los programas de prevención de $\mathrm{CaCU}$, en un escenario ideal, incluyan la vacuna contra VPH en un abordaje integral (8) y, en función de la incertidumbre existente, recomiendan que los recursos se asignen mayoritariamente a tamizaje más que a vacunación(12).

Para el caso mexicano, es pertinente considerar si puede resultar más efectivo fortalecer las acciones de detección oportuna de $\mathrm{CaCU}$ en localidades con limitado acceso a tamizaje y tratamiento y una mayor tasa de mortalidad por cáncer, y valorar la introducción de la vacuna allí donde la exposición al riesgo de contraer VPH es importante y la tasa de incidencia de infección por el virus es mayor.

\section{Población objetivo}

El plan piloto de vacunar a un porcentaje de las mujeres de 12 años plantea preguntas sobre la equidad y transparencia en la selección de la población, ya que las circunstancias asociadas a condiciones de vulnerabilidad-edad, etnicidad, condición socioeconómica o acceso a servicios de salud- justifican una política de focalización compensatoria.

La estrategia del plan piloto establece que la vacuna contra VPH se dirija de manera prioritaria y focalizada a jóvenes de comunidades con importante presencia

4 Hernández Ávila, Lazcano Ponce, Reynales Ghigematsu, Rodríguez, Myers (June 2006). Cost-effectiveness analysis of a potential human papillomavirus vaccine (HPV) and its epidemiological and economic impact on prevention and treatment of cervical cancer in Mexico. Technical report. Dossier Merck Sharpe and Dohme for request of inclusion to Mexican formulary 2006-2007.

Barr E. Updated efficacy data - Gardasil ^, HPV vaccines. Merck Research Laboratories; 2007. En prensa.

Glaxo Smith Kline Biológicos. Evaluación del costo-efectividad de un programa de vacunación para prevenir el cáncer de cuello uterino en México. Dossier GlaxoSmithKline para inclusión al Cuadro Básico de Medicamentos 2007-2008. 
indígena. La intervención se enfocaría a reducir el número de casos sin tratamiento de $\mathrm{CaCU}$ en población indígena que tradicionalmente experimenta problemas de acceso a servicios de tamizaje y tratamiento. Asimismo, contempla ofrecer la vacuna a jóvenes en comunidades urbano-marginadas, donde potencialmente se ubica la población con el mayor grado de prevalencia y alto riesgo de infección por VPH. Ambos objetivos del plan son importantes; sin embargo, se debe citar evidencia acerca de las condiciones de salud que ambas intervenciones buscan modificar.

Una política focalizada requiere establecer criterios de selección rigurosos, transparentes y basados en evidencia suficiente de que este grupo de población en particular es el que -ante la falta de recursos suficientes- debería verse beneficiado por la política. Alcanzar consenso de tal beneficio focalizado es en la práctica muy difícil.

Conocer cómo se emplean los recursos públicos para promover y proteger la salud de las mujeres considera cuestiones como las necesidades de los grupos marginados y vulnerables en la sociedad. La inclusión de la vacuna contra VPH no debería perpetuar las injusticias en salud, sino introducirse mediante estrategias bien planeadas y basadas en evidencia que permitan beneficiar a las jóvenes en mayor riesgo(13).

Para sustentar la recomendación es esencial incluir información epidemiológica que demuestre que las mujeres de estos grupos están más expuestas que otras a padecer de $\mathrm{CaCU}$ como consecuencia del virus del $\mathrm{VPH}$, que tienen menores opciones de acceso a la detección y menores oportunidades de recibir tratamiento. Si tal información no es provista existe el riesgo de que la política pública sea interpretada como paternalismo y profundice el estereotipo existente de una población indígena o urbana marginada incapaz de cuidar su propia salud.

\section{Grupo de edad que se debe vacunar}

En los escenarios planteados por el análisis de costoefectividad y sobre la base de la literatura publicada, se propone el grupo de 12 ańos por dos razones principales. Las jóvenes pueden ser suficientemente maduras para entender el propósito de la vacunación pero, la gran mayoría, aún jóvenes para su debut sexual(14) y, por lo tanto, no han estado expuestas a la infección por VPH(15). También, la vacuna contra VPH puede suministrarse junto con otras vacunas(9) por lo que podría sumarse al esquema de vacunación existente en México(16).

Las recomendaciones de vacunación basadas en la edad han demostrado ser más eficaces que las basadas en el riesgo, ya que socialmente tienen menor estigma y son más fáciles de implementar. En ocasiones, las recomendaciones relacionadas con el riesgo no funcionan, porque las personas eligen no ser identificadas con el grupo en riesgo -particularmente si se trata de una infección transmitida por contacto sexual-o no se identifican a sí mismas como parte del grupo objeto de la vacunación(17). De ahí la importancia de considerar extender la vacunación de manera universal a las mujeres de 12 ańos de edad.

Sin embargo, sería oportuno discutir la conveniencia de extender la vacunación a un rango mayor de edades y/o la implementación de programas de "repesca", buscando cubrir a mujeres mayores de 12 años que aún no han estado expuestas a variedades oncogénicas de VPH y que se pueden beneficiar con la vacuna. El rango de edades para el cual la vacuna ha sido recomendada por diversos países es de 9 a 26 años (ver tabla 1 ). Los programas de repesca pueden ser una opción que contribuya a la equidad intergeneracional, siempre que puedan ser financieramente sustentables en el corto y mediano plazo.

\section{Criterios para el financiamiento}

El antecedente que apoya el plan piloto proviene de la iniciativa del Senado de la República(1). Al analizarla, pareciera que el programa piloto responde de forma importante a la labor de cabildeo realizada por las empresas farmacéuticas productoras de la vacuna contra VPH, colocando así al sector público mexicano en una situación de desventaja para negociar un precio posible de sufragar por parte de éste. El esquema de plan piloto ya ha sido empleado con éxito por empresas farmacéuticas para la introducción de otras vacunas de nueva generación -como la antineumococcica-, con un resultado adverso para el sector público mexicano que encuentra difícil conseguir anualmente el presupuesto necesario y negociar una reducción en el precio de compra.

El escenario de vacunación universal, considerando una cobertura de $80 \%$ como meta para una intervención dirigida a adolescentes, no resultaría plausible si se 
mantienen los precios por dosis de vacuna hasta ahora ofertados (entre \$ 60 y \$ 80 dólares americanos) que hacen financieramente inviable su introducción y operación mientras no esté cercano al umbral calculado de costo-efectividad de $\$ 17$ dólares por dosis(3). Dicho precio es uno de varios componentes clave que debe considerarse antes de su introducción al sector público mexicano.

La intensa campaña de promoción de los fabricantes de la vacuna tendrá como consecuencia que, en este momento, sólo la población de los deciles de ingreso más alto tenga acceso(18). Es probable que esta situación se mantenga si el precio de la vacuna es al menos cinco veces más alto de lo que el presupuesto público en salud de México puede afrontar. Aplazar la decisión debido al alto costo de la vacuna podría traer como consecuencia aumentar la injusticia socioeconómica en salud; la política debería definirse sobre la base de criterios de salud pública y no de intereses financieros.

Los programas de vacunación de cobertura universal son un factor esencial para promover y proteger la salud pública, asumiendo que se implementan con el fin de servir al interés público(19) y que un ser humano no sólo es miembro de la comunidad sino una persona cuyos derechos deben ser respetados. Los criterios a los que se les da mayor peso en la decisión de financiar e instrumentar un plan piloto de vacunación contra VPH son importantes, ya que su permanencia y extensión depende en cierta medida de los análisis de factibilidad financiera y operativa para llevarlo a cabo.

Adicionalmente, una evaluación completa del costo del programa de vacunación contra VPH necesita incluir los costos de implementación y de la estrategia educativa dirigida a la población y a los prestadores de servicios. Ambos conceptos representan costos adicionales al presupuestar la vacuna.

\section{Estrategia de implementación}

Añadir la vacuna al tamizaje cervical sin considerar la infraestructura necesaria para brindar el servicio podría resultar en dispendio de recursos(20). Sería oportuno desarrollar un mapa del camino que recorre la vacuna desde el laboratorio hasta su aplicación de modo de identificar los elementos necesarios para su implementación exitosa, así como las brechas actualmente existentes en cuanto a recursos e infraestructura.
La puesta en marcha de un programa de prevención del $\mathrm{CaCU}$ que respete la normatividad en la materia(21) y que incluya la vacuna contra VPH será compleja. Resulta esencial una cuidadosa planeación y educación del público, de las participantes y de los prestadores de servicios de salud. Es importante que las mujeres vacunadas no interpreten el mensaje de manera inexacta y lleguen a pensar que están seguras y protegidas completamente contra el $\mathrm{CaCU}$, eligiendo abandonar el esquema regular de tamizaje(1). Los mensajes de educación en salud, adicionales y complementarios para retardar el debut sexual de los adolescentes, el uso del condón y la reducción de riesgos sobre el VPH, así como sobre el VIH, deberán ser claramente articulados y evaluar su impacto durante la eventual introducción de la vacuna(22).

Adicionalmente, el costo-efectividad de las intervenciones deberá reevaluarse considerando la respuesta de la población a la vacunación, ya que lanzar programas en poblaciones en las cuales un número significativo de potenciales receptoras de la vacuna se niega a recibirla pone en riesgo la efectividad inicialmente calculada. Es importante recordar que no se está atendiendo la demanda espontánea de pacientes enfermas, sino invitando a menores de edad sanas a someterse a una intervención de la que podrían derivarse consecuencias. Esto plantea exigencias específicas al solicitar su consentimiento para participar en la vacunación.

En este caso, hace falta desarrollar un análisis y evaluación detallada de costos, beneficios y consecuencias, tanto de la vacuna en sí misma como de la implementación del programa. En el caso del VPH, dado que es una infección de transmisión sexual, la vacuna deberá ser comparada con alternativas como la provisión de información sobre el uso de prácticas sexuales seguras.

\section{Aceptación de la vacuna}

Varios estudios han demostrado diversos grados de aceptación de la vacuna contra VPH en función de las condiciones educativas y de la información que tenga la población respecto de ella(23). Una preocupación latente en comunidades con mayor presencia indígena está relacionada con las barreras culturales que existen para la aceptación de nuevas tecnologías en salud.

Habrá que explorar la idea de que quizá es posible generar la demanda de la vacuna contra VPH en 
comunidades indígenas una vez que esta haya sido introducida en localidades urbano-marginadas -en las cuales la prevalencia de infección por VPH es mayor y ya es una necesidad identificada por la población-, de forma que disminuyan las posibilidades de que sea vista como una intervención impuesta.

\section{Riesgos percibidos}

La vacuna contra VPH puede generar inquietudes en la población por el riesgo de efectos secundarios y su impacto en el comportamiento social, particularmente la preocupación de que pueda promover la actividad sexual en la adolescencia, otorgando un falso sentido de seguridad ante los riesgos sexuales(23).

Aun cuando hasta hoy no se dispone de evidencia fehaciente sobre efectos adversos graves derivados de la vacuna contra $\mathrm{VPH}(24)$ y dado que la efectividad de la misma no ha sido probada a largo plazo, es necesario considerar quién asumirá la responsabilidad y, en su caso, establecer un sistema de compensación para las personas vacunadas que resultaren afectadas como consecuencia directa de la vacunación.

Los efectos de un programa de vacunación no se limitan a los riesgos y beneficios en salud. Se deben considerar también las molestias que se generan con la inyección, ya que un número importante de mujeres vacunadas experimentaron dolor, hinchazón y enrojecimiento(25). En lo posible, se deben minimizar estos perjuicios, no sólo porque así convenga a los individuos sino porque evitarlos contribuye a una mejor disposición para recibir la vacuna.

Serán esenciales esfuerzos coordinados en educación en salud, aun en comunidades donde la vacuna sea bien recibida, ya que existe el riesgo de que sea percibida como reemplazo del tamizaje de $\mathrm{CaCU}$ más que como una medida adicional de protección.

Otras inquietudes se centran en cuestiones de equidad de género producidas por una vacuna hasta el momento sólo accesible a mujeres, ya que la efectividad de la vacuna en hombres no ha sido establecida de manera definitiva(26). Sin embargo, algunos grupos podrían estar interesados en su potencial beneficio masculino o en el beneficio acumulado para las mujeres cuando también se vacune a los hombres. Adicionalmente, puede provocar inquietud en ciertos grupos de po- blación que la vacuna sea en realidad un esquema de esterilización encubierta(27).

Si bien un programa de vacunación sirve al interés público, no implica que todos los miembros de la población recibirán iguales beneficios. Por lo tanto, la distribución de riesgos, perjuicios y beneficios debería ser lo más justa posible. Tal necesidad se muestra aquí con un importante componente de género, porque sólo un grupo de la población soporta los riesgos y los perjuicios de una vacuna y otro grupo recibe los beneficios.

\section{Consentimiento}

Las inquietudes de la población conducen a cuestiones acerca de quién decidirá si una joven de 12 años recibirá la vacuna o no. Habrá aún más cuestiones por resolver sobre cómo y bajo qué condiciones las jóvenes y sus padres podrían decidir no participar en la vacunación(28). Algunos países y gobiernos locales han emprendido programas de vacunación obligatoria para el ingreso a la escuela; si tal tipo de política es implementada en México, se deberá evaluar la posible oposición religiosa, filosófica o ideológica que deberá ser manejada contemplando las implicaciones políticas de tal decisión(29).

Considerando la importancia que tiene la adopción autónoma de decisiones de salud por personas competentes, la participación en un programa de vacunación(30,31), universal o piloto, debe ser voluntaria, a menos que la vacuna sea esencial para prevenir un daño preciso y grave. Dado que la infección por VPH se adquiere a través de la actividad sexual y no es de tipo contagioso, no se puede argüir para su aplicación criterios similares a otras vacunas, como sarampión o tosferina. La ausencia de daño potencial a terceros limita la opción de hacer obligatoria la vacuna contra $\mathrm{VPH}$, ya que la convertiría en una política de tipo paternalista(29), inaceptable para algunos grupos de población.

\section{Información}

Si la intención es ofrecer la vacuna dentro del esquema nacional de vacunación, será crucial brindar mayor información y educar sobre sus características a las mujeres que se vacunarán y a sus padres, con el fin de que puedan tomar una decisión informada y participar de manera voluntaria. 
En un programa de vacunación voluntaria, tratándose de menores de edad, se brinda a los padres la oportunidad de elegir en nombre de sus hijos, quienes no estarán obligados a aceptar la vacuna. Es importante diseñar estrategias de educación efectivas en términos de cobertura, calidad y certeza de la información.

Debe respetarse la confianza pública puesta en los programas, ya que México cuenta con el esquema básico de vacunación más completo de América Latina, tanto por el número de vacunas que lo integran como por la población a la que beneficia(32). Para mantener dicha confianza se deben hacer esfuerzos por explicar a la población que la vacuna contra $\mathrm{VPH}$ es una medida preventiva y no terapéutica respecto al $\mathrm{CaCU}$, así como aclarar los beneficios reales y no meramente potenciales de la vacuna: contra qué tipo de infecciones protege y contra cuáles no, los riesgos asociados existentes y la duración de su protección en el tiempo.

\section{Conclusiones}

La vacuna contra VPH, así como las tecnologías para la prevención del $\mathrm{CaCU}$ nuevas y tradicionales, deben ser examinadas en el caso específico mexicano reconociendo el contexto global de carga de la enfermedad y del riesgo de cáncer, sin que se sobrestimen los resultados tangibles o potenciales de la vacuna.

Es necesario tomar medidas que aseguren que, en caso que así se decida, la prevención primaria de la vacuna estará integrada a la prevención secundaria de la detección oportuna a través de tamizaje, y tener en cuenta que la posible reducción de casos nuevos y de mortalidad por $\mathrm{CaCU}$ será el reflejo no de dos programas excluyentes sino complementarios. La posibilidad de introducir la vacuna contra VPH en los servicios públicos de salud mexicanos con criterios de equidad depende de una estrategia de negociación eficaz, para lograr una reducción de su precio cercana al umbral de costo-efectividad calculado y que pueda ser afrontado por el presupuesto en salud.

Si se decide incluir la vacuna será indispensable tener en cuenta que una estrategia de aplicación basada en la edad tendrá mayores posibilidades de éxito que la propuesta basada en el riesgo, especialmente tratándose de una intervención que puede generar impacto en los medios de comunicación, ya que involucra dos conceptos sensibles para la población en general: la transmisión por contacto sexual y la posibilidad de desarrollar cáncer.

Los resultados del análisis y la discusión sobre la vacuna en el contexto mexicano indican que, con base en la actual evidencia de su efectividad, un programa de inmunización universal dirigido a mujeres es en este momento prematuro si no se establecen estrategias efectivas de información y educación respecto de expectativas reales de protección que ofrece, ya que podría tener consecuencias negativas no previstas para las participantes y la sociedad.

Exponer la evidencia científica y los motivos para la toma de decisiones es esencial; también, dirigirse de manera responsable hacia una decisión política sobre la implementación de programas de vacunación, tamizaje y tratamiento de $\mathrm{CaCU}$, con base en el potencial para salvar vidas que tienen las tecnologías y los programas en cuestión, siempre y cuando respeten los derechos, opiniones y preocupaciones de las mujeres a las que va dirigida la vacuna y que representen un valor agregado para la sociedad mexicana en su conjunto.

Las autoras agradecen los valiosos comentarios de Jason Lakin, de la Universidad de Harvard.

\section{Referencias}

1. Senado de la República de México. LX Legislatura. Gaceta Parlamentaria 2007; 126.

2. Cámara de Diputados de México. LX Legislatura. Anexos Presupuesto de Egresos 2008. Gaceta Parlamentaria 2007; 10(2.381).

3. Gutiérrez-Delgado C, Báez-Mendoza C, González-Pier E, et al. Costo-efectividad generalizado de intervenciones preventivas contra el cáncer cervical en mujeres mexicanas: Resultados de un modelo markoviano con la perspectiva del sector público. Salud Pública México 2008, 50: 2.

4. Daniels N, Sabin JE. Setting limits fairly: Can we learn to share medical resources? Oxford: Oxford University Press; 2002.

5. FUTURE II Study Group. Quadrivalent vaccine against human papillomavirus to prevent high-grade cervical lesions. N Engl J Med 2007; 356(19): 1915-1927. 
6. Baden LR, Curfman GD, Morrissey S, Drazen JM. Human papillomavirus vaccine. Opportunity and challenge. $N$ Engl J Med 2007; 356:1990-1991.

7. Paavonen J, Jenkins D, Bosch FX, et al. Efficacy of a prophylactic adjuvanted bivalent L1 virus-like-particle vaccine against infection with human papillomavirus types 16 and 18 in young women: an interim analysis of a phase III double-blind, randomized controlled trial. The Lancet 2007; 369: 2161-2170.

8. Agosti JM, Goldie SJ. Introducing HPV Vaccine in Developing Countries. Key Challenges and Issues. N Engl J Med 2007; 356(19): 1908-1910.

9. Danish Center for Health Technology Assessment. Reduction in the risk of cervical cancer by vaccination against human papillomavirus (HPV). A health technology assessment-Summary 2007; 9(1).

10. Rambout L, Hopkins L, Button B, Fergusson D. Prophylactic vaccination against human papillomavirus infection and disease in women: a systematic review of randomized controlled trials. CMAJ 2007; 177(5): 469-479.

11. Elbasha E, Dasbach E, Insigna R. Model for assessing Human Papillomavirus vaccination strategies. Emerg Infect Dis 2007; 13(1): 28-41.

12. Suba EJ, Donnelly AD, Furia LM, et al. Cervical cancer prevention for all the world's women: genuine promise resides in skilled quality management rather than novel screening approaches. Diagn Cytopathol 2007; 35: 187-191.

13. Lippman A, Melnychuk R, Shimmin C, Boscoe M. Human papillomavirus, vaccines and women's health: questions and cautions, CMAJ 2007;177: 484-487.

14. Instituto Mexicano de la Juventud. Encuesta Nacional de Juventud 2005. Resultados Preliminares. México; 2005.

15. Department of Health and Ageing. Australian Government funding of Gardasil. Australian Government; 2006.

16. Consejo Nacional de Vacunación. Manual de procedimientos técnicos de vacunación. México: actualización 2003.

17. Humiston SG, Rosenthal SL. Challenges to vaccinating adolescents: vaccine implementation issues. The Ped Infect Dis $J$ 2005; 24(6): S134-S140.

18. Franco EL. Commentary: Health inequity could increase in poor countries if universal HPV vaccination is not adopted. BMJ 2007, 335: 378-379.

19. Coughlin S. Ethical issues in epidemiologic research and public health practice. Emerging Themes in Epidemiology 2006; 3: 16.

20. Raffle AE. Challenges of implementing human papillomavirus (HPV) vaccination policy. BMJ 2007; 335: 375-377.

21. Subsecretaría de Promoción y Prevención de la Salud. NOM-014-SSA2-1994: Sobre el control y tratamiento del cáncer cérvico-uterino. México: Secretaría de Salud, Diario Oficial de la Federación, 31 de mayo de 2007.

22. World Health Organization. Preparing for the introduction of HPV Vaccines: Policy and Programme Guidance for Countries 2006. Disponible en http://www.who.int/reproductive-health/publications/hpvvaccines/

23. Zimmerman RK. Ethical analysis of HPV vaccine policy options. Vaccine 2006; 24: 4812-4820.

24. Eggertson L. Adverse events reported for HPV vaccine, CMAJ 2007; 177(10): 1169-1170.

25. Center for Disease Control (CDC). Quadrivalent Human Papillomavirus Vaccine. Recommendations of the Advisory Committee on Immunization Practices. ACIP 2007; 56(RR02);1-24.

26. De Melo-Martin I. The promise of the human papillomavirus vaccine does not confer immunity against ethical reflection. The Oncologist 2006; 11(4): 393-396.

27. Program for Appropriate Technology in Health (PATH). Introducing HPV Vaccines in Developing Countries: Overcoming the Challenges: 2005.

28. Charo R. Politics, parents, and prophylaxis-mandating HPV vaccination in the United States. N EnglJ Med 2007; 356: 1905-1908.

29. Colgrove J. The Ethics and Politics of Compulsory HPV Vaccination. N Engl J Med 2006; 355(23): 2389-2391.

30. Verweij M, Dawson A. Ethical principles for collective immunization programmes. Vaccine 2004; 22(3): 122-3126.

31. Wilson JMG, Jungner G. Principles and Practice of screening for disease. WHO Public Health Papers 1968; Ch. 2: 26-39.

32. Secretaría de Salud de México. Información para la rendición de cuentas 2001-2005. México: 2006.

Recibido: 22 de abril de 2008

Aceptado: 25 de mayo de 2008 